\title{
Mesoporous Silica Functionalized by Cyclam-Metal Groups: Spectroscopic Studies and Numerical Modeling
}

\author{
M. Makowska-Janusik • A. Kassiba • \\ N. Errien • A. Mehdi
}

Received: 10 June 2010/ Accepted: 4 August 2010/Published online: 24 August 2010

(C) The Author(s) 2010. This article is published with open access at Springerlink.com

\begin{abstract}
Mesoporous silica functionalized by cyclammetal molecules were investigated by spectroscopic methods including Raman, IR, UV-VIS absorption and EPR technique. To analyse quantitatively the physical features, numerical models were developed using the density functional theory method. Thus, the construction of molecular geometries and their optimisation analysis were achieved on the cyclam-metal molecules in vacuum as well as constrained by the host mesoporous silica matrixes. In this context and with regard to the paramagnetic nature of the metals chelated by cyclam molecules, EPR technique allows probing the metal environments which can be compared to theoretical results inferred from numerical models. The vibrational and optical properties were exhaustively investigated and the assignment of the main features was quantitatively discussed thanks to the carried out numerical analyses. The developed approach point out the possibility to define a targeted application of such functional materials based on the possibility to fine tune the absorption features in a wide wavelength range by stabilizing defined configurations of the cyclam-metal groups.
\end{abstract}

\section{Makowska-Janusik ( $\square$ )}

Institute of Physics, Jan Dlugosz University, al. Armii. Krajowej 13/15, 42-200 Czestochowa, Poland

e-mail: m.makowska@ajd.czest.pl

\section{A. Kassiba $\cdot$ N. Errien}

Institut de Recherche en Ingénierie Moléculaire et Matériaux Fonctionnels, FR CNRS n ${ }^{\circ} 2575$ Laboratoire de Physique de l'Etat Condensé, UMR CNRS n ${ }^{\circ}$ 6087, Université du Maine, Avenue Olivier Messiaen, 72085 Le Mans Cedex 9, France

\section{A. Mehdi}

Institut Charles Gerhardt, UMR 5253 Chimie Moléculaire et Organisation du Solide, CC 1701 Université Montpellier II, Place E. Bataillon, F-34095 Montpellier Cedex 5, France
Keywords Mesoporous silica $\cdot$ Cyclam · IR spectroscopy · Raman · UV-VIS absorption · Cyclam-cooper . Cyclam-nickel

\section{Introduction}

Mesoporous silica functionalised by organometallic active groups constitute attractive architectures for a wide range of potential applications like e.g. magneto-optical switchers and bi-sensors [1-3], drug delivery controllers [4-6] or catalytic agents $[7,8]$. Thus, the considered materials are the subject of broad field of investigations [9-12] since the functionalities can be tuned by changing the nature of the functional groups such as nanoparticles [13], magnetic clusters [14], organic or organometallic molecules [15]. Besides, these materials can be prepared by incorporating several classes of organic chelates depending on the targeted property [16-19]. In this aim, the development of different synthesis routes was extensively experienced and applied to silica backbone on the form of mesoporous materials with pore sizes ranging from 2 to $20 \mathrm{~nm}$. These pores are suitable for hosting several functional vectors. Additionally, the carried out synthesis methods are able to draw innovating architectures, where the active groups are located either in the channels or constitute a part of the silica backbone [20]. A functionalization of the silica pores can be performed either by grafting the active molecules to the silica channels walls using organosilica chains or by direct synthesis methods [21]. In this case, the functional groups are expected to be more regularly distributed in the channels leading to a homogeneous media. From another side, fundamental understanding of the physical phenomena involved in such class of materials can orient and contribute to open new possibilities of applications. 
Particularly, the characteristic features of the functional materials are monitored by the intrinsic properties of the host matrices and those of the active molecules as well as the mutual interactions involved at the interfaces.

One of the promising doping agent concerns organic macrocycle group such as tetraazamacrocycle called 1,4,8,11-tetraazacyclotetradecane and referred below as cyclam. The key interest from cyclam lies in its ability to form thermodynamically stable organometallic complexes by chelating several metal ions. Their features were exploited in several area of applications [22-27] including the cyclam derivatives, which show anti-tumor activity [28], alternatively may be used as contrast agents for magnetic resonance or radio-diagnostic imaging $[29,30]$ or as therapeutic radio-pharmaceuticals [31]. Cyclam has the form of cyclic molecule formed by four imine groups acting as donor centres with the nitrogen atoms being characterized by $s p^{3}$ hybridisation. The metallic ion chelated by a cyclam is coordinated with the nitrogen atoms and located in the centre of the cycle cavity. The size and the morphology of the cavity can be changed as function of the chelated metal ions which monitor the characteristic features and activities of the organometallic group.

The present work is dedicated to investigate physical and structural properties of cyclam molecules chelating metal ions such as nickel and copper. The organometallic groups are studied solely, grafted in pores of mesoporous silica matrixes or even introduced in the mesoporous silica backbone. Spectroscopic methods (IR, Raman, UV-VIS absorption, EPR) were conjugated to shed lights on the main features of mentioned hybrid architectures. Quantitative analyses were carried out thanks to theoretical model and numerical simulation methods used as key supports for the performed experiments. Even though the doping rate of active groups in the host matrixes is in the range of 2.6-25\%, the optical and vibrational features as well as the EPR spectral parameters of the organometallic complexes were calculated and analysed using the density functional theory (DFT) computational method.

The experimental results and the carried out numerical simulations have contributed to clarify the electronic, magnetic and structural properties of cyclam derivatives associated with the host mesoporous silica matrices. The EPR experiments and the carried out analysis of the spectral line intensities and line widths have contributed to give precise insight on the local structure of the metallic ion environment [32]. These facts were also underlined by Raman and optical UV-VIS absorption experiments. Particularly, the clustering of the cyclam-ion groups significantly affects Raman spectra by a large broadening and wavenumber shifts of the corresponding bands. In this context, the performed numerical methods have contributed to point out the main parameters, which monitor the optical and vibrational features of the organometallic groups in agreement with the experimental analyses as it will be discussed in the present report.

\section{Experimental Details}

\subsection{Materials Preparation}

All reactions were carried out under argon using a vacuum line. Solvents were dried and distilled just before use. Triblock copolymer P123, 3-chloropropyltrimethoxysilane and TEOS were purchased from Aldrich and used as supplied. The cyclam was purchased from Chematech. $\mathrm{NiCl}_{2}$ powders supplied from Acros were dried before to be used.

Several routines of synthesis were developed to obtain cyclam groups chelating metal transition ions (refereed below as cyclam-metal) as well as mesoporous silica with pores or networks functionalized by cyclam-metal groups. The doping rate of the mesoporous silica by cyclam-metal molecules was performed in the range $2.6-25 \%$ with respect to the precursors of silica. The achievement of the correct organization and structure of the material requires appropriate procedures. However, the forthcoming report exemplifies in detail, the synthesis of the mesoporous silica sample functionalized in the pores by cyclam-metal groups with a doping rate about $11 \%$.

\subsubsection{Preparation of Pre-functionalized (Silica Matrix)-Cl}

This first synthesized material (silica matrix)-Cl, represents the mesoporus silica with the location of $\left(\mathrm{CH}_{2}\right)_{n}$-Cl chains dedicated to attach the functionalising groups such as cyclam-metal. In this aim, triblock copolymer $\left(\mathrm{EO}_{20}\right.$ $\mathrm{PO}_{70} \mathrm{EO}_{20}$ with $\mathrm{PEO}=$ poly(ethylene oxide) and $\mathrm{PPO}=$ poly(propylene oxide)) Pluronic P123 (4.0 g) were dissolved in $160 \mathrm{~mL}$ of an aqueous solution of $\mathrm{HCl}$ $(\mathrm{pH}=1.5)$. The resulting clear solution was added to a mixture of 3-Chloro-Propyl-Trimethoxysilane $(0.84 \mathrm{~g}$, $4.49 \mathrm{mmol}$ ) and Tetraethylorthosilicate (TEOS) (8.41 g, $40.41 \mathrm{mmol}$ ). A vigorous stirring for $3 \mathrm{~h}$ was performed at room temperature until a transparent solution appeared. Then, the solution was transferred in a hot oil bath at $60{ }^{\circ} \mathrm{C}$ and $\mathrm{NaF}$ (76.0 $\mathrm{mg}, 1.80 \mathrm{mmol}$ ) was immediately added to induce the polycondensation. A white precipitate appeared within a few minutes and the resulting suspension was further stirred for 2 days at $60{ }^{\circ} \mathrm{C}$. The resulting white powder was filtered off and the surfactant was selectively removed by soxhlet extraction over ethanol for $24 \mathrm{~h}$. After drying at $120{ }^{\circ} \mathrm{C}$ overnight under vacuum, $2.89 \mathrm{~g}(94 \%)$ of (silica matrix)-Cl (defined SBA-Cl) were obtained as white powder. 


\subsubsection{Preparation of (Silica Matrix)-Cyclam}

This step consists in realisation of mesoporous silica functionalized by cyclam groups. The process uses a mixture of 1,4,8,11-tetraazacyclotetradecane (cyclam) (0.42 g, 2.09 $\mathrm{mmol})$, SBA-Cl (1.00 g, $1.49 \mathrm{mmol})$ dissolved in $90 \mathrm{~mL}$ of acetonitrile and triethylamine $(1.1 \mathrm{~g}, 10.9 \mathrm{mmol})$. The used mixture was heated under reflux $\left(78^{\circ} \mathrm{C}\right)$ and stirring for 2 days. The white solid was quantitatively recovered by filtration and washed five times with hot chloroform and three times with hot ethanol to remove the excess of cyclam. After drying at $120^{\circ} \mathrm{C}$ overnight under vacuum, $1.10 \mathrm{~g}$ (95\%) of mesoporus silica functionalized by cyclam molecules (SBA-cyclam) were obtained as a white powder.

\subsubsection{Preparation of (Silica Matrix)-Cyclam-Ni}

The final step realizes the mesoporous silica functionalized by organometallic groups which consist in cyclam molecules chelating nickel ions. This was achieved using $1.00 \mathrm{~g}$ (1.49 mmol) of SBA-cyclam dissolved in an ethanolic solution of $\mathrm{NiCl}_{2}(0.017 \mathrm{M}, 123 \mathrm{~mL})$. The resulting suspension was heated overnight under reflux with stirring. The solid was quantitatively recovered by filtration and washed with dry ethanol several times to remove the excess of nickel salt. After drying at $120{ }^{\circ} \mathrm{C}$ overnight under vacuum $1.08 \mathrm{~g}$ (97\%) of SBA-cyclam chelating nickel, referred below as SBA-cyclam$\mathrm{Ni}$, the investigated material was obtained as a green powder.

Schematic summary of the carried out synthesis is shown in Fig. 1. By this way the mesoporus silica pores or networks were functionalised by cyclam-metal groups. With the same procedure, the functionalization of mesoporous silica (SBA) by cyclam-copper groups was achieved. In the last step of procedure discussed above, the ethanol solution of $\mathrm{CuCl}_{2}$ instead of $\mathrm{NiCl}_{2}$ was used.

\subsection{Characterization Methods}

\subsubsection{Transmission Electron Microscopy}

The TEM observations were carried out in order to check the homogeneity and morphology of the mesoporous silica. JEOL 2010 equipment with an acceleration voltage of $200 \mathrm{kV}$ was used as well as liquid nitrogen anticontamination trap devoted to improve the vacuum around the sample. All TEM images were taken by using a contrast diaphragm. The sample preparation consists in small amount of powder, grinded in pure ethanol. One droplet of the suspension was then deposited on copper grid covered by amorphous carbon membrane. At first glance, the sample resists quite well to the electron beam and elongated grains were clearly seen as illustrated in Fig. 2. The morphology of the mesoporous grains is marked by alternating clear and dark fringes with a periodicity about $10 \mathrm{~nm}$. Such contrast reveals the porous structure of the sample with parallel and well aligned pores. However, with regard to the functional cyclam-Ni group dimensions, it was not easy to evaluate their presence in the host silica.

\subsubsection{Spectroscopic Investigations}

Room temperature UV-VIS diffuse reflectivity spectra were collected on a finely grinded sample with a Cary $5 \mathrm{G}$
Fig. 1 Structure of investigated materials: (a) mesoporous silica in hexagonal lattice (SBA-15), (b) mesoporous silica functionalized by cyclam groups chelating nickel ions inside the pore, (c) mesoporous silica functionalized by cyclam groups chelating nickel ions in the network
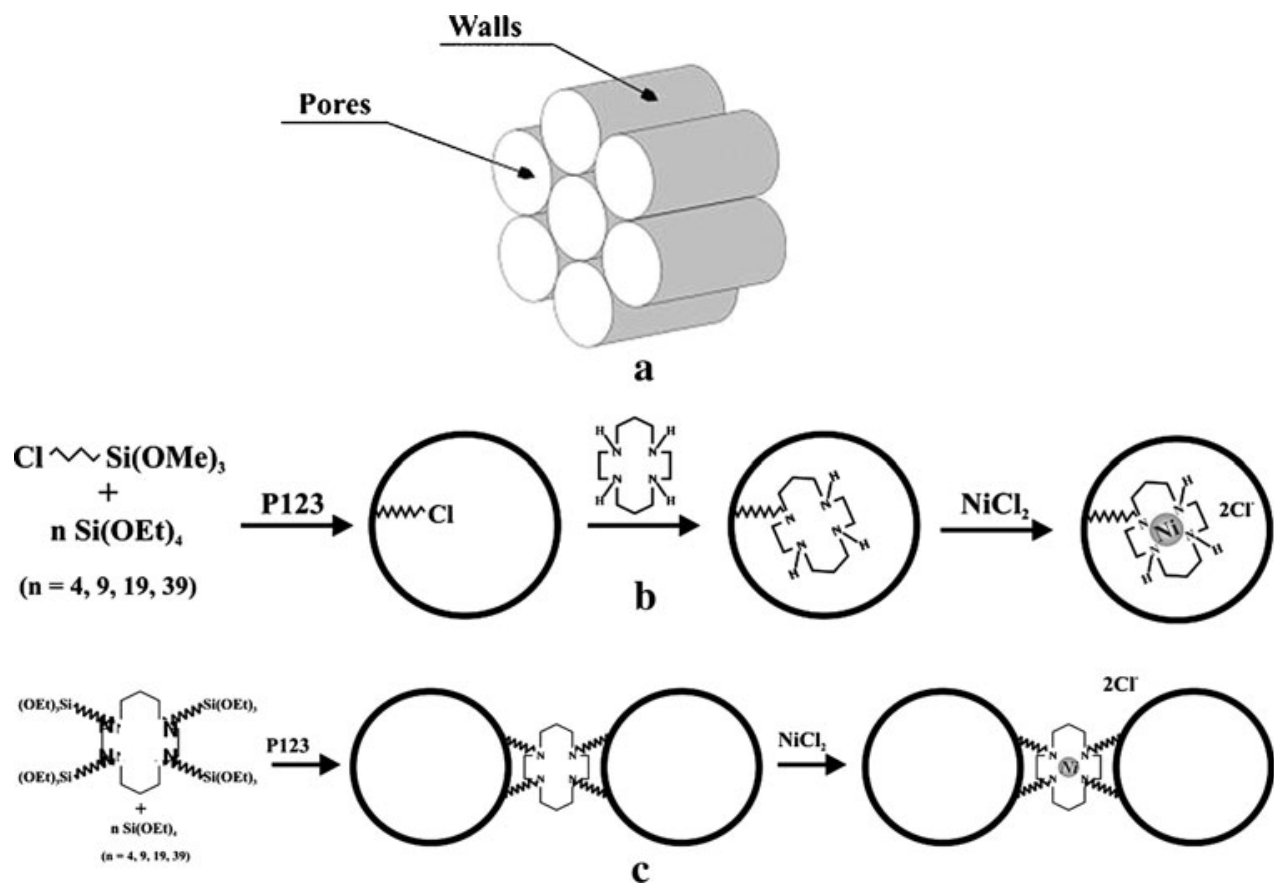

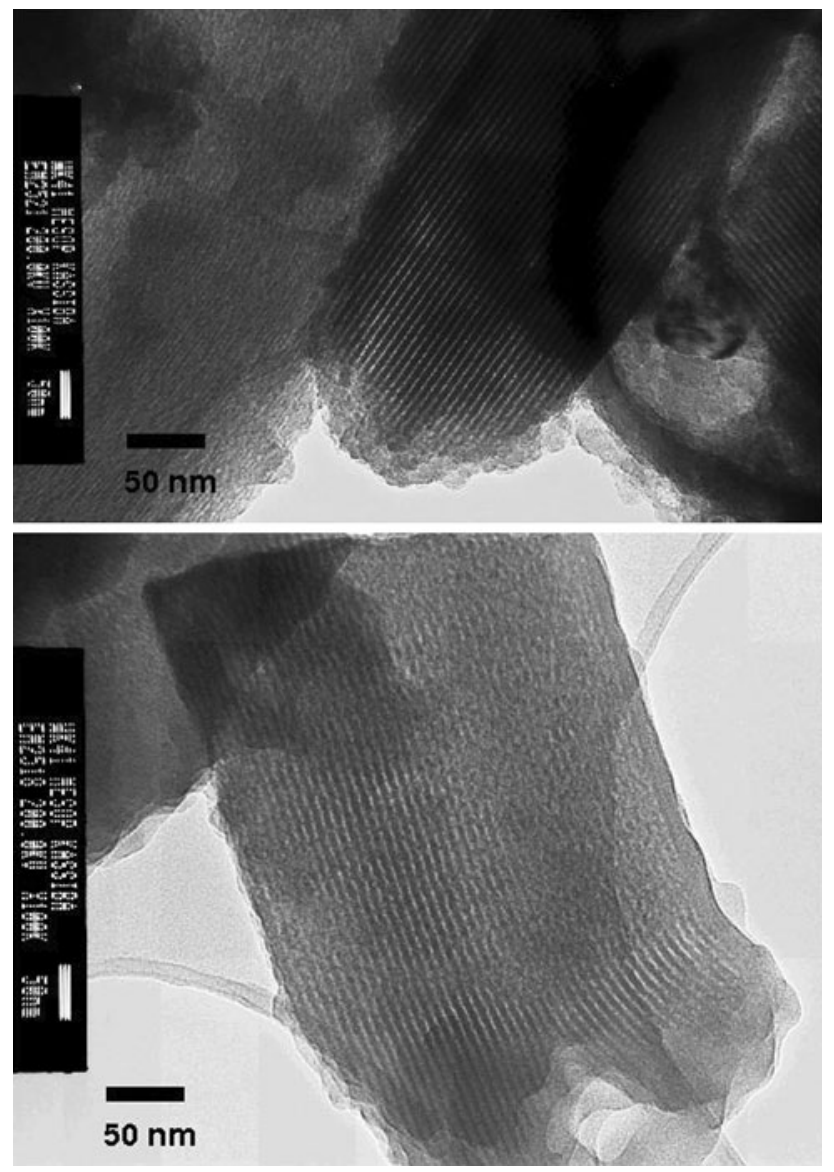

Fig. 2 TEM images of functionalised mesoporous silica doped in the pores

spectrometer (Varian). The instrument was equipped with a Cary Praying Mantis Diffuse Reflectance accessory and computer control using the Scan software. The absorption in diffuse reflectance versus wavelength measurements were made in the 250-800 $\mathrm{nm}$ range with a $2 \mathrm{~nm}$ step using a mirror as reference (100\% reflectance).

The infrared absorption measurements were performed using Perkin-Elmer FTIR spectrometer model 2000. The samples were prepared by grinding the samples with $\mathrm{KBr}$ and to form cold pressed pellets.

A confocal micro-Raman spectrometer coupled with an $\mathrm{Ar}^{+}$laser was used at room temperature. The experiments were realized by selecting the $514 \mathrm{~nm}$ or the $647 \mathrm{~nm}$ radiations with an incident power about $40 \mathrm{~mW}$. The experimental conditions are kept the same for all investigated samples. Finally, for the paramagnetic cyclam-metal molecules, the EPR investigations were performed and the characterisation of the key features related to mesoporous silica functionalised by cyclam-metal groups were completed [32].

\section{Numerical Simulation Models}

The computer simulations were performed with the aim to ensure a key support for the experimental investigations performed by structural and spectroscopic means on the functional materials. The numerical models consider several configurations of the cyclam molecules related to nonchelating macrocycle as well as to nickel and copper chelated molecules with defined valence and coordination features. Indeed, with regard to the stability of the cyclammetal molecular structure, bonding of the central metal transition ion with chlorine ions must be considered. This lead to pyramidal-like environment for copper while bi-pyramidal-like local environments holds for cyclamnickel. The size of the investigated systems requires the use of the density functional theory (DFT) formalism to fully optimize their geometries in vacuum without any symmetry constraint.

The proposed calculations were performed with ADF program package [33] at the Generalized Gradient Approximation (GGA) [34]. The BLYP exchange-correlation potential was used as equivalent to the gradient correction proposed by Becke for the exchange part of potential [35] and for the Lee-Yang-Parr correlation correction [36-38]. All atoms were described within the triple$\zeta$ (TZ) basis set defined in the program database. The core electrons such as $1 s-3 p$ for $\mathrm{Cu}$ and $\mathrm{Ni}, 1 s-2 p$ for $\mathrm{Cl}, 1 s$ for $\mathrm{C}$ and $\mathrm{N}$ were kept frozen for the considered atoms. The calculations performed at the BLYP level and employing the TZ basis set, were found to be relevant for the geometry optimization in the case of molecular structures based on metals of the second and third raw of the transition elements in the periodic table [39]. The correct feature of the optimized structure which ensures local minimum in the potential energy surface, was confirmed by vibrational frequency computations. In all cases the absence of negative frequencies confirmed that the optimized geometries correspond to stable configurations.

The numerical methods carried out on cyclam-metal groups were devoted to simulate the spectral parameters experimentally obtained from Raman, IR and UV-VIS absorption as well as the EPR spectra. The vibrational frequencies were determined from the normal modes related to the molecule in its electronic ground state and within the harmonic approximation. The IR frequencies were calculated numerically using GEOMETRY module in ADF program [40, 41]. Time dependent DFT (TDDFT) computations at the equilibrium geometry were employed as an accessory tool in the assignment of the electronic and Raman spectra [42] while the calculations of the Raman frequencies were performed using the RESPONSE module [43] of the ADF program package. In the computation 
process dedicated to IR and Raman frequencies, the same basis sets and the relevant exchange-correlation potentials were used as for the geometry optimisation procedure. Finally, the EXCITATION module implemented in ADF program was employed to calculate the electronic excitation of the valence electrons with regard to the UV-VIS absorption spectra [43-46]. Particularly, Davidson diagonalization procedure [47] was considered for the excited states calculations implementing the BLYP potential and the TZ2P basis set.

\section{Results and Discussion}

\subsection{Geometry of the Cyclam-Metal Complexes}

DFT calculations were performed on organometallic groups as isolated objects or grafted on silica walls. Even if the structures of isolated molecules were developed earlier on similar composition [48], the approach developed in the present work examines the specific cases of the cyclammetal groups and consider constrained molecules due to the host mesoporous silica. Indeed, cyclam molecules chelating metal ions and grafted inside the silica pores or embedded into the silica backbone alter significantly both vibrational and optical properties as well.

For the isolated cyclam molecules, DFT calculations were performed and compared successfully to molecular geometries involved in crystals [49]. This fact supports the relevance of DFT method to draw reliable analysis for the structural peculiarities of cyclam molecules chelating metal ions. As mentioned in the paragraph related to computational details, the full geometry optimizations for all molecules were performed based on the criterion of minimization of the second derivative of the energy on the pseudopotential surface supported by the search for positive eigenvalues of the Hessian. The relevant data are summarized in Table 1.

According to this numerical analysis, the copper ion in cyclam- $\mathrm{Cu}$ structure is fully coordinated by the tetra-aza sub-units in the configuration of a square geometry. This fact contrasts with the case of nickel even if the radius of both ions is comparable as well as the expected Jahn-Teller nature which characterizes both ions. In the cyclam-Ni complex, the four imine groups are not equivalent and this seems to be connected with the nickel electronic configuration. Indeed, former experimental reports on the cyclamNi complexes state on the less constrained geometries, when planar configurations hold and stabilize low-spin for Nickel ions [50,51]. However, high-spin state is compatible with a geometry configuration as trans-octahedral complex [52, 53]. Noticeably, the macrocycles exhibits versatile geometries for cyclam-metal and its derivatives while the stable valence of the metal ions impose the bonding of the central atom to chlorine. As example, cyclam-Ni and cyclam-Cu were considered with a bond metal-Cl out from the square planar geometry formed by imine groups. Optimization of the geometries leads to nearly the same configuration but the

Table 1 Structural data obtained by theoretical optimization of cyclam molecule chelated metal ion, where $\mathrm{R}=\mathrm{Ni}, \mathrm{Ni}-\mathrm{Cl}, \mathrm{Ni}-\mathrm{Cl} \mathrm{C}_{2}, \mathrm{Cu}$ and $\mathrm{Cu}-\mathrm{Cl}$

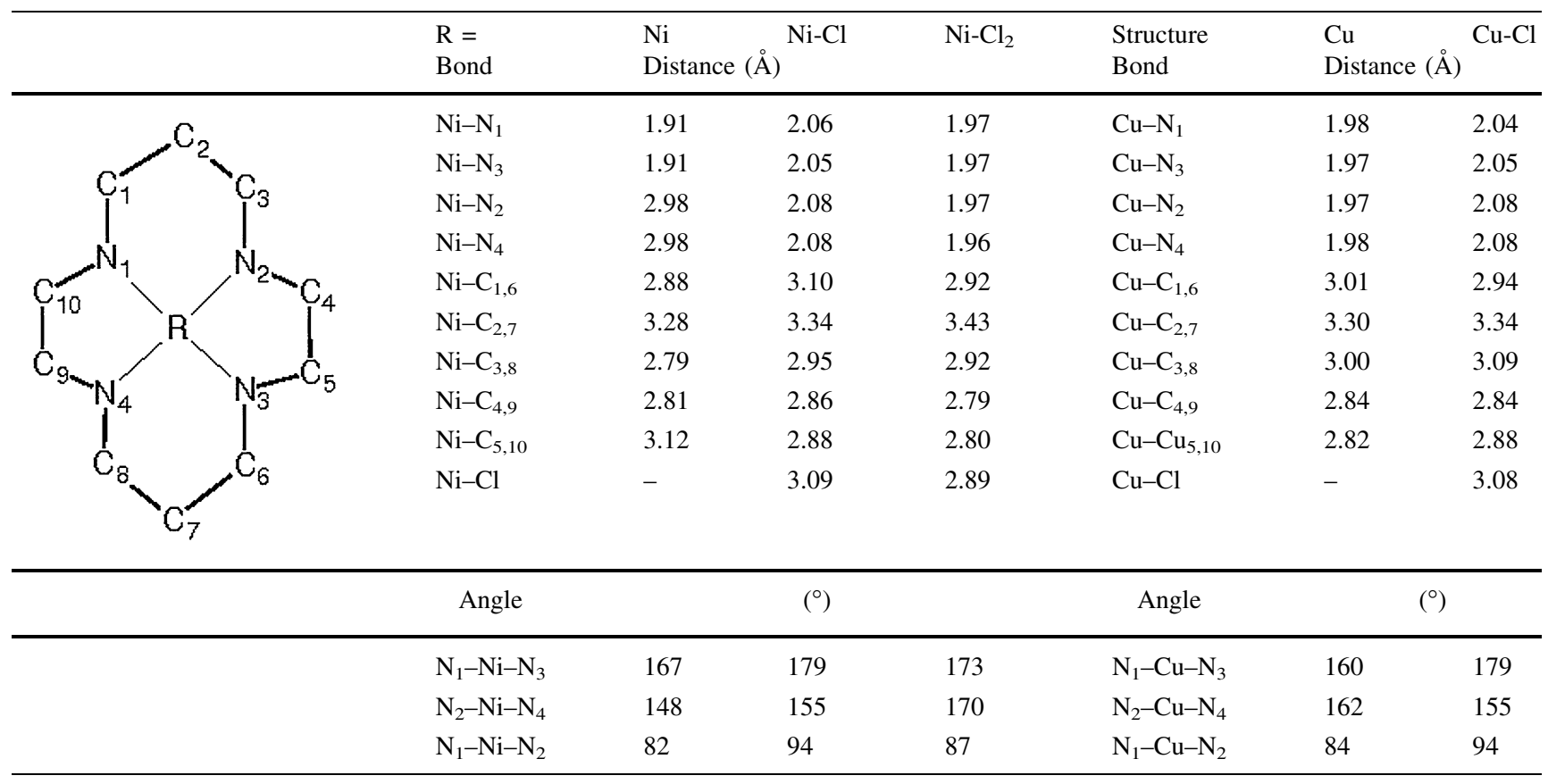


total energy of cyclam-Cu with $\mathrm{Cu}-\mathrm{Cl}$ complex is lower than in the case of nickel based complexes. Alternatively, bonding of the central nickel with two chlorines seems more stable from the total energy criterion and can be compared to experimental reports based on X-ray diffraction [54]. The nickel-chlorine bonds were fond anomalously longer than usually shown for undistorted octahedral complexes. As matter of fact, the numerical analysis yields a stable geometry in the form of tetragonal coordinated nickel with square planar bonds between nickel ion and imine groups and two perpendicular $\mathrm{Ni}-\mathrm{Cl}$ bonds. The carried out calculations of the $\mathrm{Ni}-\mathrm{Cl}$ bonds exhibit larger lengths than the experimentally determined ones while the angles defined in Table 1 are in very good agreement with the experimental values related to the same structures. It is worth noting that the departure of the bond lengths about $0.4 \AA$ is a consequence of the main drawback of the used BLYP approximation in the considered numerical model. The reported discrepancy is justified by the tendency of mentioned approximation to overestimate the bond lengths as was generally reported for environments with transition metal ions based compounds [55].

\subsection{EPR Probing of Ligand Fields}

EPR spectroscopy is a very sensitive to the local environments around paramagnetic ions. For the cyclam- $\mathrm{Cu}$ and cyclam-Ni geometries, the EPR signal of copper and nickel ions are well resolved. The involved interactions can be identified from the anisotropy of the EPR spectra or from the hyperfine or super-hyperfine structure which originates respectively from the nuclear spins of copper nuclei or that of chlorine ions. Typical EPR signals related to cyclam-Cu and cyclam-Ni molecules grafted in the mesoporous silica channels are shown in Fig. 3. From the EPR signal of copper (Fig. 3a), it seems that only weakly resolved hyperfine interaction can be shown but a large anisotropy of the magnetic interaction seems to be involved. In the case of nickel spectrum (Fig. 3b), the anisotropy is very low compared to the well resolved hyperfine structure, which testifies that chlorine ions are involved in the first coordination shell of nickel ions. Quantitatively, the analysis of the EPR spectra for the considered cases is performed by the following spin-hamiltonian:

$H=\beta \vec{B} \cdot \tilde{g} \cdot \vec{S}+\sum_{i} \vec{S} \cdot \tilde{A}_{i} \cdot \vec{I}_{i}$

where $S=1 / 2$ represents the effective electronic spin of $\mathrm{Cu}^{2+}$ and $\mathrm{Ni}^{3+}$. The first term of equation is related to the Zeeman interaction with the magnetic $\tilde{g}$ tensor being dependent on the local environment and symmetry of the paramagnetic ions. The second term can be either associated to an interaction between electronic spin or nuclear

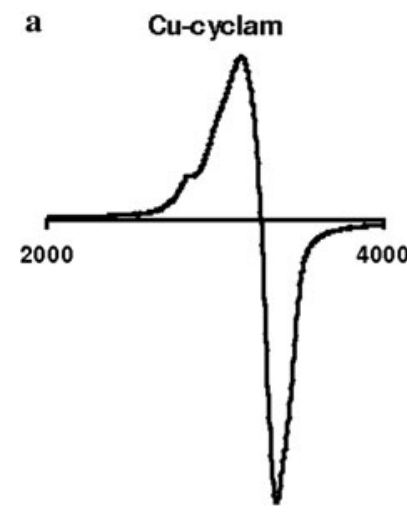

Magnetic field (Gauss)

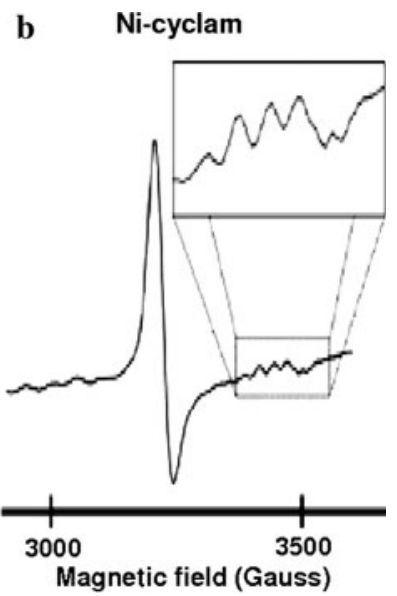

Fig. 3 EPR spectra of copper and nickel ions in the organometallic cyclam-metal groups used to functionnalize mesoporus silica

spin of copper nuclei $(i=1)$ as well as to the interactions between the electronic spin and the $i$ th neighboring chlorines ion.

An adjustment of the EPR spectrum shapes allow to obtain the spectral EPR parameters summarized in Table 2 for copper and nickel ions chelated by cyclam molecules, respectively. The EPR results are then consistent with the anisotropy of the magnetic environments involved for both ions as well as an axial symmetry, which can be understood by planar coordination with imine groups and perpendicular bonding of the metal to chlorine ions.

Thus, for the copper ions, the $g$-tensor components are theoretically described in an axial environments by:

$g_{\|}=2.0023+8 k_{\mathrm{Cl}} \frac{\left|\lambda_{0}\right|}{\Delta_{\|}}$
$g_{\perp}=2.0023+2 k_{\mathrm{N}} \frac{\left|\lambda_{0}\right|}{\Delta_{\perp}}$

where $k_{\mathrm{Cl}}$ and $k_{\mathrm{N}}$ represents the covalence parameters of the $\mathrm{Cu}-\mathrm{Cl}$ and $\mathrm{Cu}-\mathrm{N}$, respectively. The $\lambda_{0}$ is the spin-orbit coupling constant, which contributes to the energies of $d$ orbitals of the $\mathrm{Cu}^{2+}$ ion and $\Delta_{\perp, \|}$ characterize the energy splitting between electronic $d$ states.

The experimental value of $g$-tensor components are consistent with the fundamental orbitals associated with $d_{x^{2}-y^{2}}$ and covalence parameters between copper and

Table 2 EPR spectral parameters for cyclam-Cu and cyclam-Ni molecules grafted in the silica channels. The $A_{x, y, z}$ represent the hyper fine parameters for copper ions while for Nickel they arc related to super-hyperfine coupling with axial chlorine ions

\begin{tabular}{llllrrr}
\hline Cyclam-R- $i$ & $g_{x}$ & $g_{y}$ & $g_{z}$ & $A_{x}$ & $A_{y}$ & $A_{z}$ \\
\hline $\mathrm{R}=\mathrm{Cu}^{2+}$ & 2.070 & 2.070 & 2.210 & 20 & 20 & 170 \\
$\mathrm{M}=\mathrm{Ni}^{3+}, i=\mathrm{Cl}(1), \mathrm{Cl}(2)$ & 2.178 & 2.178 & 2.0025 & 0 & 0 & 28 \\
\hline
\end{tabular}


nitrogen $\left(k_{\mathrm{Cu}-\mathrm{N}}\right)$ and between copper and chlorine $\left(k_{\mathrm{Cu}-\mathrm{Cl}}\right)$

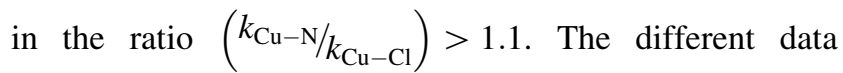
inferred from EPR investigations suggest, that as far as the first coordination shell of copper is concerned, pyramidal like coordination of copper is realized and consist in planar bonding with the imine groups and in perpendicular direction of elongated $\mathrm{Cu}-\mathrm{Cl}$ bond compared to the planar $\mathrm{Cu}-\mathrm{N}$ ones. This analysis supports the stability of the pyramidal like environment of cyclam-Cu quantitatively demonstrated by numerical modeling.

The same analysis carried out for the cyclam-Ni groups shows that the EPR signal is consistent with the valence of $\mathrm{Ni}^{3+}$ interacting with two chlorines ions. This result is in agreement with the numerical model which indicates a more stable and energetically favorable the tetragonal coordination of the nickel ions.

\subsection{Raman Investigations of Cyclam-Ni in Mesoporus Silica}

\subsubsection{Experiments}

The Raman spectra were recorded at room temperature for the samples described in Sect. 2.1. Bare cyclam-Ni powders or cyclam-Ni molecules grafted in the silica pores give rise to the high wavenumber Raman spectra depicted in Fig. 4 for different doping rates $(2.6 \%, 5.3 \%, 11.1 \%)$. Whatever the synthesis method (direct or two steps process) or the concentration of cyclam-Ni molecules in the silica channels, the Raman spectra exhibit similar features. The main difference exists only between cyclam-Ni in bare powder form and in the silica. For the isolated molecules, the Raman spectrum shows quite resolved and narrow bands in contrast to the broad features observed when these molecules are in the silica pores.

In the high wavenumber range, the Raman shifts from $2,830 \mathrm{~cm}^{-1}$ to $2,960 \mathrm{~cm}^{-1}$ can be assigned to symmetric and asymmetric stretching vibration of the $\mathrm{CH}_{2}$ groups. Precisely, the weak bands located at $2,837 \mathrm{~cm}^{-1}$ and $2,860 \mathrm{~cm}^{-1}$ result from symmetric $\mathrm{CH}_{2}$ stretching [56]. The large band located at $2,923 \mathrm{~cm}^{-1}$ can be assigned to asymmetric stretching of $\mathrm{CH}_{2}$ groups [57] while the shift at $2,959 \mathrm{~cm}^{-1}$ is attributed to vibrations of $\mathrm{CH}_{2}$ groups [58]. $\mathrm{N}-\mathrm{H}$ stretching mode are characterized by the vibrational features observed around $3,250 \mathrm{~cm}^{-1}$, but the splitting on the corresponding band in two components $\left(3,230 \mathrm{~cm}^{-1}\right.$ and $3,260 \mathrm{~cm}^{-1}$ ) is an indication of non equivalency between the local environment of the bonded hydrogen atoms [59]. A tentative explanation for such splitting can be suggested from the computational work performed on cyclam-Ni molecules reported in Table 1. Indeed, two molecular configurations are involved and consist in the

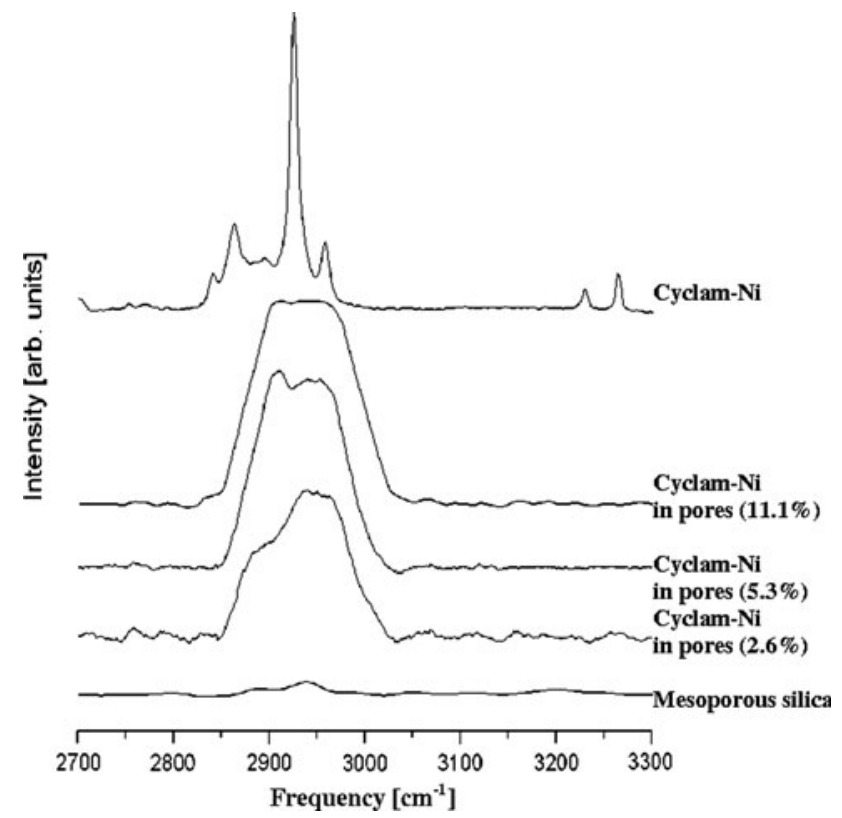

Fig. 4 High wavenumber range of Raman spectra obtained for mesoporous silica with cyclam groups inside pores. The Raman spectra for the cyclam groups alone and pure silica are also reported for comparison

bonding of the metal to one or two chlorines. Even if the last configuration $\left(\mathrm{Ni}-\mathrm{Cl}_{2}\right)$ is energetically more favorable, the existence of $\mathrm{Ni}-\mathrm{Cl}$ groups is relevant and can contribute to the shift of the $\mathrm{N}-\mathrm{H}$ vibrational band.

In the low wavenumber range $\left[300-1,000 \mathrm{~cm}^{-1}\right]$, the multiple Raman bands from the silica backbone did not allow a consistent comparison with the functionalized silica channels by cyclam-metal molecules. So, this part of analysis was not considered in the present report.

\subsubsection{Numerical Simulation of Raman Frequencies}

The numerical simulations of the Raman spectra were performed in order to assign quantitatively the origin of the observed Raman bands limited for our purpose to the high wavenumber side of the spectra. The simulation data are carried out on the mesoporous silica functionalized by cyclam or cyclam-Ni complexes with two configurations of bonds between nickel and chlorine ions ( $\mathrm{Ni}-\mathrm{Cl}$ and $\left.\mathrm{Ni}-\mathrm{Cl}_{2}\right)$. The calculated characteristic wavenumbers are summarized in Table 3 and compared with the main experimental data. The first column of Table 3 reports the position of experimental Raman bands obtained for the cyclam-Ni molecules. The three remaining columns contain the theoretical Raman band positions for different configurations of cyclam and cyclam-Ni complexes. The forth, dashed column with bold numbers report the average theoretically obtained wavenumber for different cyclam or cyclam-Ni complexes. The assignment of the Raman bands to defined vibrational modes 
Table 3 Experimental and simulated peaks position of Raman spectra

\begin{tabular}{llll}
\hline $\begin{array}{l}\text { Experiment } \\
\left(\mathrm{cm}^{-1}\right)\end{array}$ & $\begin{array}{l}\text { Cyclam- } \\
\mathrm{NiCl}_{2}\left(\mathrm{~cm}^{-1}\right)\end{array}$ & $\begin{array}{l}\text { Cyclam }+ \\
\left.\mathrm{NiCl}\left(\mathrm{cm}^{-1}\right)\right]\end{array}$ & $\begin{array}{l}\text { Cyclam } \\
\left(\mathrm{cm}^{-1}\right)\end{array}$ \\
\hline
\end{tabular}

316

347

359

385

407

427

453

476

503

520

545

570

583

727

774

800

843

865

886

909

930

966

994

1,011

1,032

1,055

1,067

1,087

1,097

1,108

1,131

1,152

1,173

1,182

1,201

1,228

1,251

\section{9}

343

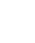

353

365

390

406

430

449

475

503

521

560

583

731

970

1,034

1,048

1,079

1,104

1,127

1,165

1,158

1,190

1,208

1,230

1,252
Table 3 continued

\begin{tabular}{|c|c|c|c|c|c|}
\hline \multirow{2}{*}{$\begin{array}{l}\text { Experiment } \\
\left(\mathrm{cm}^{-1}\right)\end{array}$} & \multicolumn{2}{|c|}{$\begin{array}{l}\text { Cyclam- } \\
\mathrm{NiCl}_{2}\left(\mathrm{~cm}^{-1}\right)\end{array}$} & \multirow[t]{2}{*}{$\begin{array}{l}\text { Cyclam }+ \\
\left.\mathrm{NiCl}\left(\mathrm{cm}^{-1}\right)\right]\end{array}$} & \multirow[t]{2}{*}{$\begin{array}{l}\text { Cyclam } \\
\left(\mathrm{cm}^{-1}\right)\end{array}$} & \\
\hline & 1,258 & 1,263 & & & \\
\hline & 1,268 & & & & \\
\hline \multirow[t]{2}{*}{1,286} & 1,292 & & 1,274 & & 1,282 \\
\hline & & & 1,279 & & \\
\hline 2,841 & & & 2,839 & & \\
\hline 2,863 & & & 2,860 & & \\
\hline 2,886 & & & 2,881 & & \\
\hline \multicolumn{6}{|l|}{2,895} \\
\hline \multirow[t]{2}{*}{2,925} & 2,920 & & 2,910 & 2,926 & $2,924.5$ \\
\hline & 2,937 & & 2,925 & & \\
\hline \multirow[t]{4}{*}{2,957} & 2,956 & & 2,954 & 2,955 & 2,955 \\
\hline & 2,976 & & 2,961 & 2,978 & \\
\hline & 2,990 & & 2,975 & & \\
\hline & 3,001 & & 2,995 & 3,012 & \\
\hline \multirow[t]{2}{*}{3,230} & 3,215 & 3,230 & & & \\
\hline & 3,245 & & & & \\
\hline 3,265 & & & 3,259 & & \\
\hline
\end{tabular}

798.5
825

850

863

880

937

\section{1,000}

1,005

1,044

1,050

1,098

1,120

1,186

1,199

1,252 of given molecular bonds cannot reproduce the shape of the experimental Raman spectra because of its broadening, 843.7 probably caused by the host matrixes, where cyclam-Ni $\mathbf{8 6 4 . 7}$ groups are grafted to silica walls. To analyze quantitatively 887.7 the effect of the host silica matrix on the Raman band 909.5 broadening in the high wavenumber region, quantum chemical calculations were carried out by using the same numerical procedure as described above, modifying the boundary conditions. To take into account the interaction between the cyclam groups and the silica matrix, a constraint

1,013.5 was imposed at the atomic sites where chemical bonding exists between cyclam molecules and the silica wall. On the other hand, multiple configurations such as non-functionalized cyclam, or cyclam-Ni with $\mathrm{Ni}-\mathrm{Cl}$ or $\mathrm{Ni}-\mathrm{Cl}_{2}$ bonding are presumably involved. Also, combined vibartional features from different molecular configurations were used to tentatively reproduce the experimental spectra in the high wavenumber side as depicted in Figs. 5 and 6.

\subsection{Experimental and Numerical Analysis of IR Spectra}

Figure 7 reports the IR spectra of cylam-Ni molecules as well as the mesoporous silica with the channels being funtionalized by cyclam-Ni and cyclam-Cu groups. Narrow absorption peaks are observed for isolated cyclam-Ni molecules while broad bands with some details characterize the functionalized silica. In the case of isolated cyclam-Ni molecules the 


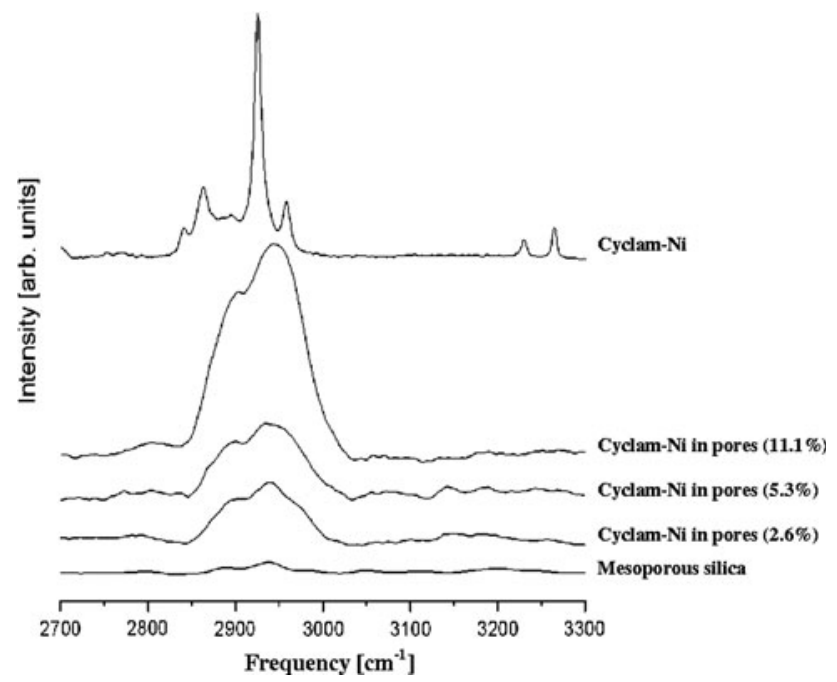

Fig. 5 High frequency part of Raman spectra of mesoporous silica with active cyclam group inside channels

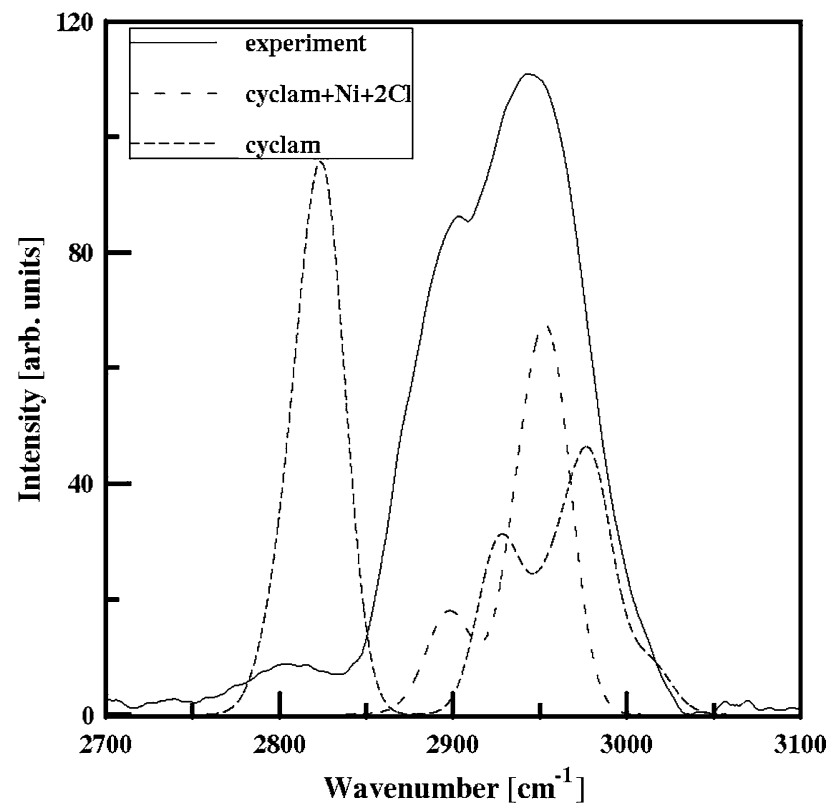

Fig. 6 High frequency part of Raman spectra of mesoporous silica with active cyclam group inside channels (solid line) measured experimentally and simulated Raman spectra for constrained pure cyclam molecule and doped with $\mathrm{NiCl}_{2}$

resolved band around $3,250 \mathrm{~cm}^{-1}$ is associated to $\mathrm{N}-\mathrm{H}$ stretching modes leading to the two observed peaks [60]. It is well-know that the strength of the hydrogen bond can be correlated with the magnitude of the shift of $\mathrm{N}-\mathrm{H}$ with respect to the free volume around them. The peaks between 2,600 and $3,000 \mathrm{~cm}^{-1}$ are consistent with the cyclam $\mathrm{C}-\mathrm{H}$ bands [61]. The intense bands observed in the $800-1,500 \mathrm{~cm}^{-1}$ region are consistent with the stretching vibration modes of metal-Cl bonds [62-64]. Finally, the peaks around $420 \mathrm{~cm}^{-1}$ correspond to vibration of $\mathrm{Cu}-\mathrm{N}$ bonds [65].

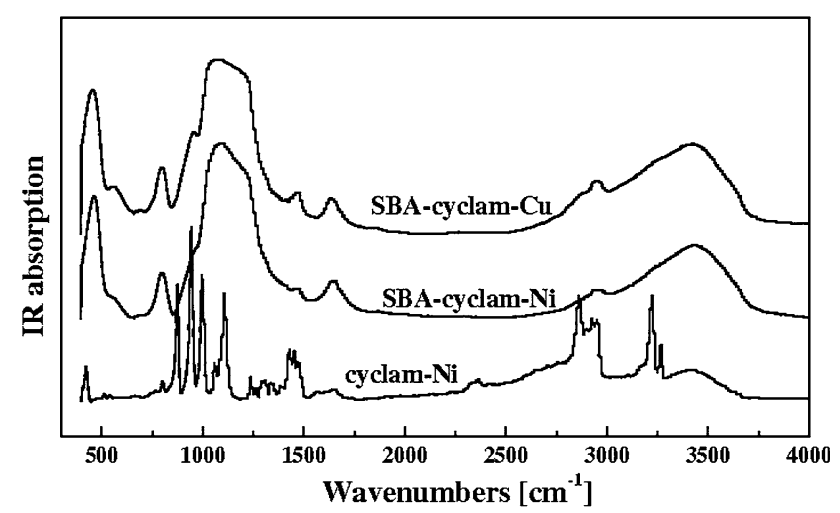

Fig. 7 Experimental IR absorption spectra obtained for cyclam chelating nickel molecules, cyclam chelating nickel and cooper complexes deposited into silica matrix

To sum up, the cyclam-Ni IR spectrum is marked by substructures in the range of $800-1,500 \mathrm{~cm}^{-1}$. Additionally, the high wavenumber side is marked by two bands. Each of them being composed of narrow components. Such features are related to isolated molecules but cannot be resolved on the IR spectra of functionalised silica irrespective to the nature of the chelated metal. Thus, a loss of information from IR studies is inferred and its consequence lies in the impossibility of IR spectroscopy to probe correctly the achievement of functionalization of silica pores by cyclam-metal group. However, the numerical approach is quite relevant to quantitatively account for the experimental IR spectra of isolated cyclam-metal molecules as exemplified on cyclam-Cu (Fig. 8).

\subsection{UV-VIS Spectroscopy}

\subsubsection{Cyclam-Nickel Molecules}

Mesoporous silica functionalized by cyclam-Ni groups gives rise to quite broad UV-VIS absorption spectra without informative details (Fig. 9a). These features characterize

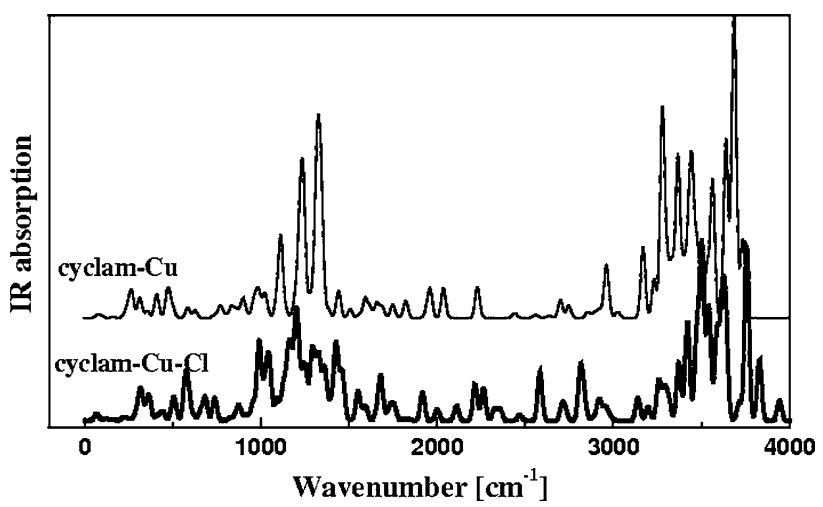

Fig. 8 IR spectra calculated for isolated cyclam-Cu molecules with or without bonding of the central metal to chlorine ion 
two samples referred as SA and SB, prepared as described in Sect. 2.1 by two steps synthesis process and a direct method, respectively. Only diffuse details can be seen around $250 \mathrm{~nm}, 377 \mathrm{~nm}$ and $470 \mathrm{~nm}$ in the functionalized silica while the bare mesoporus sample did not show any features in their absorption band in the $250-800 \mathrm{~nm}$ region. This means that the details in Fig. 9a are related to cyclam molecules chelated metal ions. Calculations of the theoretical absorption bands of cyclam-Ni molecules were carried out and the main results are summarized in Fig. 9b. Thus, it is shown that the pure cyclam molecules exhibit an absorption band near $250 \mathrm{~nm}$ while cyclam-Ni bonded to one and two chlorine have characteristic bands at $320 \mathrm{~nm}$ and $450 \mathrm{~nm}$, respectively. These results support the possibility of coexistence of bare cyclam molecules and cyclam$\mathrm{Ni}$ geometries with the central metal atom bonded to one or two chlorines ( $\left.\mathrm{Ni}-\mathrm{Cl}, \mathrm{Ni}-\mathrm{Cl}_{2}\right)$ as it was suggested also in Raman investigations part. These facts underline the possibility to tune the UV-VIS absorption in the cyclam-Ni based material by a stabilization of relevant configurations defined by the number of bonded chlorine ions. As illustrated in Fig. 9c, the obtained curve with diamonds consists of a superposition of contributions about $89.29 \%$ from cyclam- $\mathrm{NiCl}_{2}, 1.78 \%$ of cyclam-NiCl and $8.93 \%$ pure cyclam without complexation by nickel ions. The performed simulations may reproduce the shape of experimentally obtained UV-VIS absorption peaks seen in Fig. 9a.

On the other hand, though the calculated wavelength positions are shifted with respect to the measured ones. Among others it is the consequence of the chosen calculation method. However, the numerical procedure seems sound and relevant with regard to the fact that the calculations consider free and isolated molecules in vacuum contrast with a real powder, where molecular interactions are involved. Furthermore, when the active molecules are grafted inside the mesoporous silica, constraints on the molecular configurations are induced as well as a clustering which occurs in the case of inhomogeneous media as it was demonstrated by EPR investigations [32].

\subsubsection{Cyclam-Copper Molecules}

A similar approach to the case of cyclam-Ni was developed to analyze the UV-VIS absorption spectra of cyclam-Cu molecules. However, according to early report [66], copper chelated by macrocyclic complexes such as cyclams is marked by $d-d$ electronic transition leading to an absorption in the range 300-700 $\mathrm{nm}$ depending on the cooper-ligand environment [67]. As example, cyclam-Cu gives rise to an absorption band reported at $\lambda_{\max }=500 \mathrm{~nm}[68,69]$. The nature of the used solvent alters slightly the absorption band

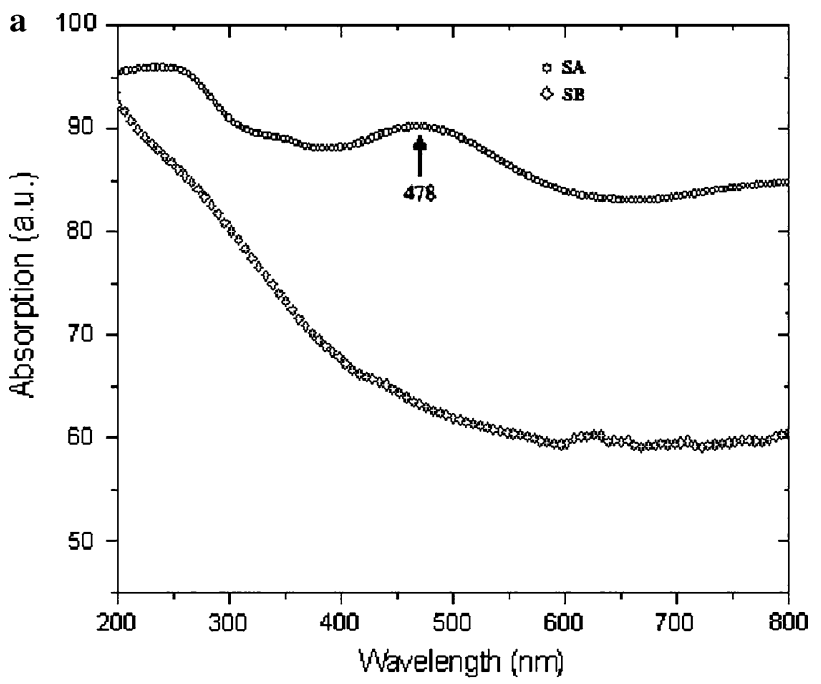

b

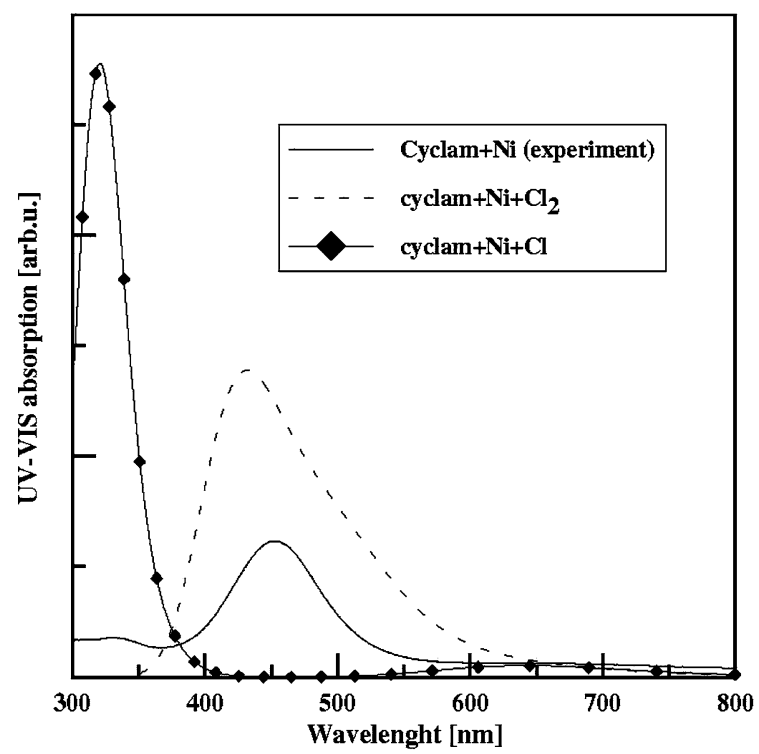

c

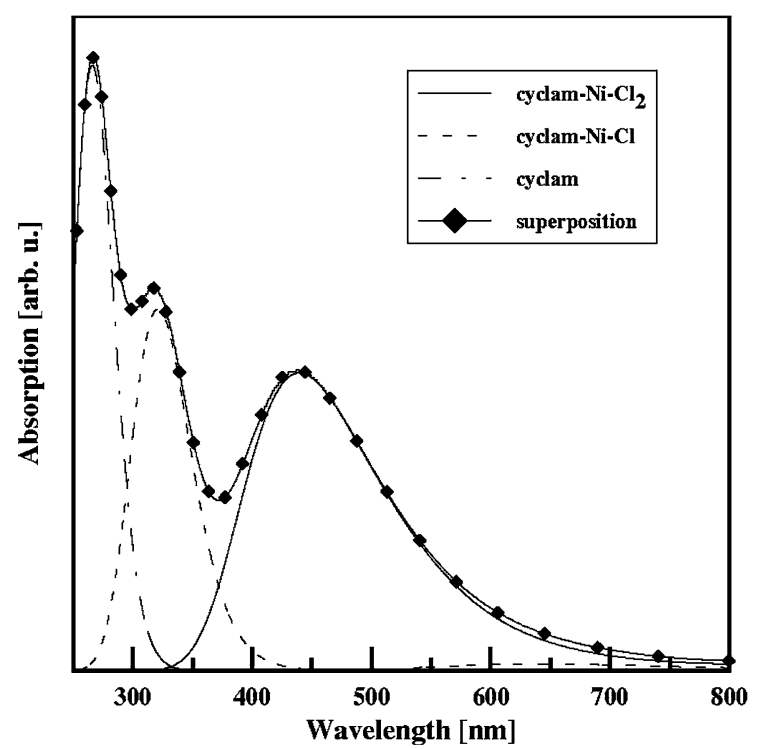


4 Fig. 9 UV-VIS absorption spectra for isolated cyclam-Ni complexes: experimental data (a), experimental and calculated spectra obtained for different configuration of $\mathrm{Ni}$ atoms (b), fitted theoretically obtained curve (line with diamonds) reproducing experimental data $(\mathbf{c})$

due to the high stability of such complexes. The first absorption band of cyclam-Cu molecules measured in methanol is at the position of $506 \mathrm{~nm}$, but the red-shift of UV-VIS peaks is noticed for the long bridging connection between macrocyclic group and host silica matrix [70]. For our purpose, the experimental UV-VIS spectra of cyclam molecules chelating copper ions were performed in water solution (Fig. 10). Changing the solvent from water to other compound e.g. methanol does not affect the shape nor the position of the absorption band. The electronic spectrum of cyclam-Cu complex in a square-planar form [71-74] or distorted-octahedral geometry exhibits a quite broad absorption band between 540 and $600 \mathrm{~nm}$, as it is shown in Fig. 10. Particularly, the experimental UV-VIS absorption spectrum details are quite understood when the two main separate contributions are taken into account. One part of the spectrum is related to bare cyclam without any metal complexation while the second contribution is related to cyclam-Cu groups. Such behavior was also observed for the cyclam-Ni based batches and seems to indicate a general features of cyclam complexes obtained by the carried out synthesis method described in Sect. 2.1.

As a main behavior demonstrated from the UV-VIS absorption experiments, it is worth noting that a coexistence of several configurations of cyclam-metal groups

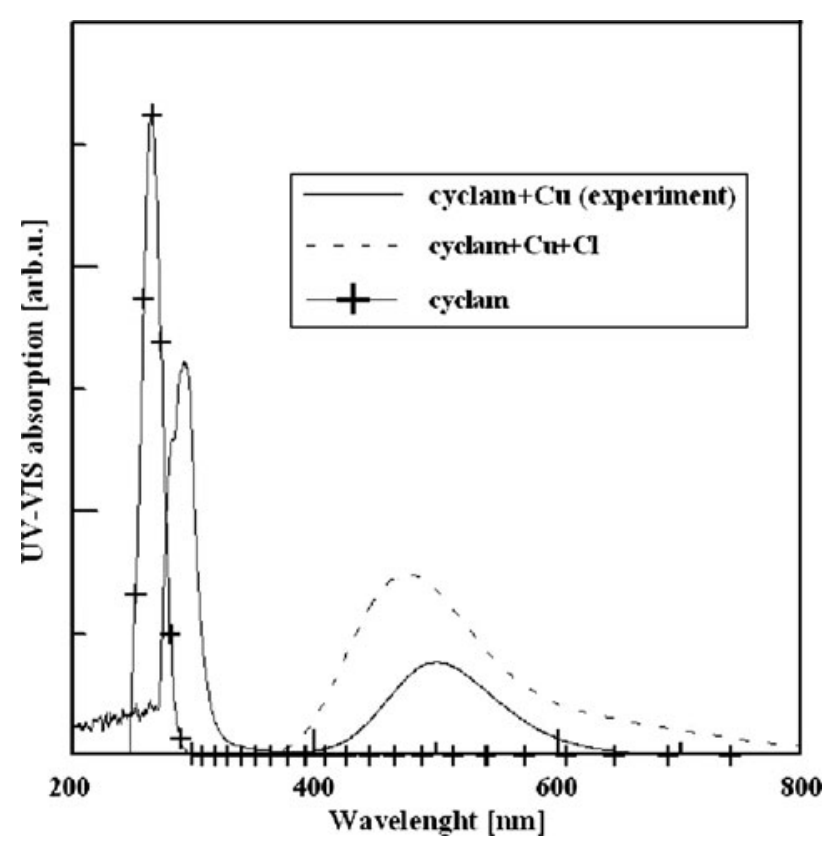

Fig. 10 Experimental and theoretical UV-VIS spectra obtained for cyclam- $\mathrm{Cu}$ complexes and their bonding with chlorine atoms seems to realize irrespective feature to the nature of the used metal. The details of the UV-VIS absorption spectra are not so informative on the location of cyclam-metal groups grafted on the silica walls; i.e. their distribution or agglomeration in the host mesoporous media. However, the numerical methods have demonstrated their relevance to correctly account all details related to bare batches of cyclam-metal with the same pertinence for both nickel and copper as well.

\section{Conclusion}

Mesoporous silica functionalised by cyclam-nickle and cyclam-cooper groups are synthesized and investigated by Raman, IR and UV-VIS absorption spectroscopy. EPR experiments have completed this set of key features and inform mainly on the local environment of metal chelated by cyclam molecules. Numerical methods have been developed to determine the local geometry of the organometallic groups, their vibrational and optical properties. Exhaustive assignment of the details on the Raman, IR and UV-Vis absorption spectra was achieved. As a common feature of the functionalized mesoporus silica by cyclammetal molecules one may conclude that different configurations of the cyclam-metal and their bonding with chlorine ions coexist with different ratios. The experimental results and the carried out numerical simulations assign correctly the physical origin of the experimental details. This concerns the local structure of cyclam-metal molecules, the thermodynamic stability of the different adopted configurations as well as the optical and vibrational features. The conducted complementary experiments contributed to draw sound diagnostic on the relevant properties of the functional materials. In this context, EPR experiments and analyses of the spectral line intensities and line widths were performed. They contribute to give precise insight on the local structure of the metallic ion environments in agreement with the carried out numerical modelling of the molecular structures. The Raman spectra combined with the numerical data inferred from DFT calculations show the relevance of the approach to analysis judiciously the peculiarities of vibrational properties when organometallic complexes are constrained by the silica walls. The UV-VIS absorption experiments have contributed to define the characteristic absorption bands and the possibility to fine tune their spectral features by stabilizing a given cyclam-metal configuration. This is of a particular importance for the possibility to combine the magnetic behaviour of cyclam-metal molecules with the optical features for magneto-optical effects; aspects which are currently under scope. 
Acknowledgment This work has benefited from the support of cooperation through Polonium program under the auspice of French MAE and Polish Ministry of Science and Higher Education authority.

Open Access This article is distributed under the terms of the Creative Commons Attribution Noncommercial License which permits any noncommercial use, distribution, and reproduction in any medium, provided the original author(s) and source are credited.

\section{References}

1. I. Slowing, B.G. Trewyn, S. Giri, V.S.-Y. Lin, Adv. Fun. Mat. 17, 1225 (2007)

2. H. Takeda, M. Ohashi, T. Tani, O. Ishitani, S. Inagaki, Inorg. Chem. 49, 4554 (2010)

3. J. Zhou, J. He, G. Li, T. Wang, D. Sun, X. Ding, J. Zhao, S. Wu, J. Phys. Chem. C 114, 7611 (2010)

4. Q. Tang, Y. Xu, D. Wu, Y. Sun, Chem. Lett. 35, 474 (2006)

5. X. Ji, Q. Hu, J.E. Hampsey, X.Q. Qiu, L. Gao, J. He, Y. Lu, Chem. Mater. 18, 2265 (2006)

6. C. Lei, P. Liu, B. Chen, Y. Mao, H. Engelmann, Y. Shin, J. Jaffar, I. Hellstrom, J. Liu, K.E. Hellstrom, J. Am. Chem. Soc. 132, 6906 (2010)

7. J.M.D. Consul, C.A. Peralta, E.V. Benvenutti, J.A.C. Ruiz, H.O. Pastore, I.M. Baibich, J. Mol. Catal. A 246, 33 (2006)

8. S.B. Hartono, S.Z. Qiao, J. Liu, K. Jack, B.P. Ladewig, Z. Hao, G.Q.M. Lu, J. Phys. Chem. C 114, 8353 (2010)

9. S. Zamani, M. Chiesa, V. Meynen, Y. Xiao, B. Prelot, J. Zajac, F. Verpoort, P. Cool, S. Van Doorslaer J. Phys. Chem. C 114, 12966 (2010)

10. R.J.P. Corriu, Eur. J. Inorg. Chem. 5, 1109 (2001)

11. B. Boury, R.J.P. Corriu, Chem. Commun. 8, 795 (2002)

12. F.N. Gu, F. Wei, J.Y. Yang, Y. Wang, J.H. Zhu, J. Phys. Chem. C 114, $8431(2010)$

13. Y. Deng, Y. Cai, Z. Sun, J. Liu, C. Liu, J. Wei, W. Li, C. Liu, Y. Wang, D. Zhao, J. Am. Chem. Soc. 132, 8466 (2010)

14. D. Zhang, X. Wang, Z. Qiao, D. Tang, Y. Liu, Q. Huo, J. Phys. Chem. C 114, 12505 (2010)

15. S. Brandes, G. David, C. Suspene, R.J.P. Corriu, R. Guilard, Chem. Eur. J. 13, 3480 (2007)

16. R.J.P. Corriu, E. Lancelle-Beltran, A. Mehdi, C. Reye, S. Brandes, R. Guilard, Chem. Mater. 15, 3152 (2003)

17. J.-M. Barbe, G. Canard, S. Brandes, R. Guilard, Angew. Chem. 117, 3163 (2005)

18. A.M. Klonkowski, B. Grobelna, T. Widernik, A. JankowskaFrydel, W. Mozgawa, Langmuir 15, 5814 (1999)

19. T. Kishida, N. Fujita, K. Sada, S. Shinkai, Langmuir 21, 9432 (2005)

20. R.J.P. Corriu, J. Organomet. Chem. 686, 32 (2003)

21. R.J.P. Corriu, A. Mehdi, C. Reye, J. Organomet. Chem. 689, 4437 (2004)

22. R.M. Izatt, K. Pawlak, J.S. Bradshaw, R.L. Bruening, Chem. Rev. 91, 1721 (1991)

23. R.M. Izatt, K. Pawlak, J.S. Bradshaw, Chem. Rev. 95, 2529 (1995)

24. M. Meyer, V. Dahaoui-Gindrey, C. Lecompte, R. Guilard, Coord. Chem. Rev. 178, 1313 (1998)

25. H. Elias, Coord. Chem. Rev. 187, 37 (1998)

26. L. Fabbrizzi, M. Lichelli, P. Pallavicini, D. Sacchi, Supramol. Chem. 13, 569 (2001)

27. K.E. Kristian, W. Song, A. Ellern, I.A. Guzei, A. Bakac, Inorg. Chem. 49, 7182 (2010)

28. J.W. Sibert, A.H. Cory, J.G. Cory, Chem. Commun. 2, 154 (2002)
29. P. Caravan, J.J. Ellison, T.J. McMurry, W.H. Lauffer, Chem. Rev. 99, 2293 (1999)

30. C.J. Anderson, M.J. Welch, Chem. Rev. 99, 2219 (1999)

31. W.A. Volkert, T.J. Hoffman, Chem. Rev. 99, 2269 (1999)

32. L. Laskowski, A. Kassiba, M. Makowska-Janusik, A. Mehdi, A. Gibaud, N. Errien, J. Swiatek, J. Phys.: Condens. Matter 21, 076004 (2009)

33. G. te Velde, F.M. Bickelhaupt, S.J.A. van Gisbergen, C. Fonseca Guerra, E.J. Baerends, J.G. Snijders, T. Ziegler, J. Comp. Chem. 22, 931 (2001)

34. M. Grüning, O.V. Gritsenko, S.J.A. van Gisbergen, E.J. Baerends, J. Chem. Phys. 116, 9591 (2002)

35. A.D. Becke, Phys. Rev. A 38, 3098 (1998)

36. C. Lee, W. Yang, R.G. Parr, Phys. Rev. B 37, 785 (1988)

37. B.G. Johnson, P.M.W. Gill, J.A. Pople, J. Chem. Phys. 98, 5612 (1993)

38. T.V. Russo, R.L. Martin, P.J. Hay, J. Chem. Phys. 101, 7729 (1994)

39. B.A. Hess Jr., J.P. Cross, H.C. Manning, D.J. Bornhop, J. Chem. Phys. 123, 244302 (2005)

40. L. Fan, T. Ziegler, J. Chem. Phys. 96, 9005 (1992)

41. L. Fan, T. Ziegler, J. Am. Chem. Soc. 114, 10890 (1992)

42. S.J.A. van Gisbergen, Molecular Response Property Calculations Using Time Dependent Density Functional Theory, in Chemistry (Amsterdam, Vrije Universiteit, 1998)

43. S.J.A. van Gisbergen, J.G. Snijders, E.J. Baerends, Comp. Phys. Commun. 118, 119 (1999)

44. S.J.A. van Gisbergen, F. Kootstra, P.R.T. Schipper, O.V. Gritsenko, J.G. Snijders, E.J. Baerends, Phys. Rev. A 57, 2556 (1998)

45. Ch. Jamorski, M.E. Casida, D.R. Salahub, J. Chem. Phys. 104, 5134 (1996)

46. R. Bauernschmitt, R. Ahlrichs, Chem. Phys. Lett. 256, 454 (1996)

47. E.R. Davidson, J. Comput. Phys. 17, 87 (1975)

48. L. Smentek, B.A. Hess Jr., J.P. Cross, H.C. Manning, D.J. Bornhop, J. Chem. Phys. 123, 244302 (2005)

49. K.R. Adam, I.M. Atkinson, L.F. Lindoy, Inorg. Chem. 36, 480 (1997)

50. L.Y. Martin, L.J. DeHayes, L.J. Zompa, D.H. Busch, J. Am. Chem. Soc. 96, 4046 (1974)

51. Y. Hung, L.Y. Martin, S.C. Jackels, A.M. Tait, D.H. Busch, J. Am. Chem. Soc. 99, 4029 (1977)

52. L.Y. Martin, C.R. Sperati, D.H. Busch, J. Am. Chem. Soc. 99, 2968 (1977)

53. V.J. Thorn, J.C.A. Boeyens, G.J. McDougall, R.D. Hancock, J. Am. Chem. Soc. 106, 3198 (1984)

54. B. Bosnich, T.L. Tobe, G.A. Webb, Inorg. Chem. 4, 1109 (1965)

55. P.J. Hay, R.L. Martin, J. Chem. Phys. 109, 3875 (1998)

56. V.B. Singh, A.K. Singh, A.K. Rai, A.N. Singh, D.K. Rai, Spectrochim. Acta A 67, 687 (2007)

57. O. Frank, J. Jehlicka, H.G.M. Edwards, Spectrochim. Acta A 68, $1065(2007)$

58. J. Xu, I.S. Butler, D.F.R. Gibson, I. Stangel, Biomateria1s 8, 1653 (1997)

59. R. Smierciak, J. Passariello, E.L. Blinn, Inorg. Chem. 16, 2646 (1977)

60. K. Nakamoto, Infrared and Raman Spectra of Inorganic and Coordination Compounds, 3rd edn. (Wiley, New York, 1978)

61. H. Dodziuk, R.A. Kolinski, B. Korybut-Daszkiewicz, Spectrochim. Acta A 29, 511 (1973)

62. B.J. Hathaway, A.E. Underhill, J. Chem. Soc. 11, 3091 (1961)

63. K. Sakata, T. Odamura, Y. Kanbara, T. Nibu, M. Hashimoto, A. Tsuge, Y. Moriguchi, Polyhedron 17, 1463 (1998)

64. G. Diaz, R.E. Clavijo, M.M. Campos-Vallette, M. Saavedra, S. Diez, R. Munoz, Vib. Spectrosc. 15, 201 (1997)

65. M.M.C. Vallete, in $N_{4}$-Macrocyclic Metal Complexes, ed. by J.H. Zagal, F. Bedioui, J.P. Dodelet (Springer, Berlin, 2006) 
66. A. Kassiba, M. Makowska-Janusik, J. Alauzun, W. Kafrouni, A. Mehdi, C. Reye, R.J. Corriu, A. Gibaud, J. Phys. Chem. Sol. 67, 875 (2006)

67. S. Ueki, J. Yamauchi, Inorg. Chim. Acta 338, 13 (2002)

68. M. Boiocchi, M. Bonizzoni, L. Fabbrizzi, F. Foti, M. Licchelli, A. Poggi, A. Taglietti, M. Zema, Chem. Eur. J. 10, 3209 (2004)

69. K. De Buysser, G.G. Herman, E. Bruneel, S. Hoste, I. van Driessche, Chem. Phys. 315, 286 (2005)

70. H. Stephan, G. Geipel, D. Appelhans, G. Bernhard, D. Tabuani, H. Komber, B. Voit, Tetrahedron Lett. 46, 3209 (2005)
71. H. Aneetha, Y.H. Lai, S.C. Lin, K. Panneerselvam, T.H. Lu, C.S. Chung, J. Chem. Soc. Dalton Trans. 2885 (1999)

72. C. Bucher, E. Duval, E. Espinosa, J.M. Barbe, J.N. Verpeaux, C. Amatore, R. Guilar, Eur. J. Inorg. Chem. 4, 1077 (2001)

73. A.E. Goeta, J.A.K. Howard, D. Maffeo, H. Puschmann, J.A.G. Williams, D.S. Yufit, J. Chem. Soc. Dalton Trans. 1873 (2000)

74. K. Sakata, S. Wada, N. Sato, M. Kurisu, M. Hashimoto, Y. Kkato, Inorg. Chim. Acta 119, 111 (1986) 